\title{
Promoción del trabajo colaborativo mediante la integración de las tecnologías digitales en el contexto escolar
}

\author{
Manuel Baltodano Enríquez
}

Asesor Nacional de Capacitación y Desarrollo Educativo en el Departamento de Investigación, Desarrollo e Implementación de la Dirección de Recursos Tecnológicos en Educación, Ministerio de Educación Pública, Costa Rica. manuel.baltodano.enriquez@mep.go.cr

\section{Resumen}

Este ensayo presenta algunas estrategias para el desarrollo de proyectos pedagógicos mediante la promoción del trabajo colaborativo con el apoyo de las tecnologías digitales. Durante varios años, el Departamento de Investigación, Desarrollo e Implementación de la Dirección de Recursos Tecnológicos en Educación del Ministerio de Educación Pública de Costa Rica, ha promovido diversas iniciativas para potenciar ambientes de aprendizaje colaborativo entre educadores y estudiantes, mediante el aprendizaje en red y el intercambio intercultural de estudiantes de distintas zonas del país. Todo lo anterior tiene sentido, cuando los proyectos educativos tienden a la resolución de problemas comunitarios y a la atención de los intereses de los actores curriculares involucrados. Desde la perspectiva anterior, se propone el desarrollo de proyectos pedagógicos a partir de cinco fases: la preparación, la planificación, la implementación, la evaluación y la divulgación. Con estas fases, se espera generar expectativas para el desarrollo psicomotor, socioafectivo y cognitivo de los actores curriculares que participan en los proyectos.

Palabras clave: tecnologías digitales, trabajo colaborativo, proyectos pedagógicos, comunicación digital, ambientes de aprendizaje, aprendizaje en red.

\section{Abstract \\ Promotion of the collaborative work through the integration of digital technologies in the school context}

Some strategies for developing of educational projects, based on collaborative learning using digital technologies, are discussed in this paper. For several years the Department of Research, Development and Implementation of Management Technology Resources in Education of the Ministry of Public Education of Costa Rica, has promoted various initiatives to enhance collaborative learning environments between educators and students. The main idea of these iniciatives is to create learning networks and to promove intercultural exchange among people. All this makes sense when educational projects tend to solve community problems and include it as a part of the curriculum. From this perspective, the educational projects involve five phases: a) preparation, b) planning, c) application, d) evaluation and f) dissemination, which contribute to develop emotional and cognitive capacities.

Key words: digital technologies, collaborative work, pedagogical projects, digital communication, learning environments, learning network. 


\section{INTRODUCCIÓN}

Las nuevas generaciones requieren el desarrollo de diversas competencias, orientadas a la formación de personas capaces de enfrentar los retos que la sociedad demanda. Es fundamental que los docentes estén al día con los avances del conocimiento y con las innovaciones educativas que se generan alrededor del mundo, para el mejoramiento de los procesos de enseñanza-aprendizaje, con las posibilidades educativas que ofrecen las Tecnologías Digitales (TD).

En los últimos años, en los sistemas educativos de América Latina, la incorporación de las TD, se ha dado en forma paulatina, permitiendo resolver relativamente el acceso a la educación o potenciando los aprendizajes, a la vez modificando las estrategias didácticas de los docentes; sin embargo, se cuenta con poco conocimiento o con información muy limitada, de los impactos que dicha incorporación produce en los contextos escolares, como ella mejora las habilidades adaptativas. Asimismo, los cambios tecnológicos del siglo XXI generan necesidades en la comunidad estudiantil.

En el marco de las ideas anteriormente descritas y con el mandato de promover la incorporación de las TD en los diferentes niveles del sistema educativo costarricense, el Departamento de Investigación Desarrollo e Implementación de la Dirección de Recursos Tecnológicos en Educación del Ministerio de Educación Pública de Costa Rica, ha desarrollado proyectos pedagógicos orientados al mejoramiento de la calidad de la educación, implementando diversas actividades que coadyuvan a dinamizar el currículo por medio del uso de TD; además la generación de entornos colaborativos de aprendizaje entre docentes y estudiantes.

\section{DESARROLLO DEL TEMA}

Actualmente, los avances de las TD, la globalización, los nuevos actores educativos, los entornos virtuales de enseñanza-aprendizaje y la innovación, han desdibujado y transformado los paradigmas educativos contemporáneos, impulsando la simplificación y masificación de procesos de producción, transferencia, desarrollo e implementación de conocimientos (Asociación Nacional de Universidades e Instituciones de Educación Superior, 2004). Así pues, si se observa de manera prospectiva, en relación con la evolución de los sistemas educativos globales, en los próximos años habrá un incremento cualitativo y cuantitativo de los usos y aplicaciones de las TD, específicamente, en el desarrollo de competencias adaptativas por medio de los ambientes virtuales de aprendizaje y redes sociales. Es así como, existe la necesidad de gestionar en la educación nuevos modelos pedagógicos, que permitan el acceso y la flexibilización de oportunidades de formación a diversos actores sociales, aprovechando las posibilidades que ofrece la tecnología, especialmente las redes sociales y el e-learning.

El uso de las TD con fines educativos no es ninguna novedad, su penetración se remonta a la década de los 20 del siglo pasado, con la utilización de recursos audiovisuales diversos, entre los que se destacó el uso del cine. A lo largo del siglo transcurrido, otros medios como la televisión o recursos como los multimedios, se fueron incorporando a la gama de herramientas didácticas.

En relación con lo anteriormente descrito, Claro (2010) sostiene que existen experiencias exitosas que definen buenas prácticas, centradas en la mejora de los procesos de aprendizaje, la innovación pedagógica y la organizacional. Por otra parte, las condiciones de cambio esencial fueron la orientación pedagógica del profesor, que permitió formas diferentes de aprender y diseños efectivos de clase: la infraestructura y los recursos, así como, las reformas curriculares para la promoción de las TD.

Se puede evidenciar en la vida diaria que las TD, son un motor que ha generado grandes transformaciones en la sociedad actual y la educación no está aislada de este proceso de cambios que, exige la 
sociedad del conocimiento. Por tanto, las instituciones deben fortalecer su equipo docente y su infraestructura en pro de afrontar dichos cambios.

De acuerdo con Carneiro, Toscano, y Díaz (2009), las instituciones educativas deben de transformarse en tres aspectos: el primero que permita evolucionar de una educación vista como industria, a una educación basada en un servicio de proximidad con los estudiantes, por ser ellos quienes representan un recurso central en los procesos educativos. Dejando de ver al estudiante como un sujeto pasivo y convirtiéndolo en un sujeto activo y responsable como principal artífice de su propio conocimiento. Acercándose también, a la realidad propia de cada uno de los individuos que se están formando.

En relación con el segundo aspecto, el centro educativo debe cambiar el concepto de la misión de las escuelas, de escuelas que enseñan por escuelas que aprenden, promoviendo un desarrollo integral de la persona, contemplando y garantizando aspectos fundamentales como son: aprender a ser, aprender a conocer, aprender a hacer, aprender a vivir juntos. Y procurando mejorar día a día mediante la capacitación constante.

Por último, como tercer aspecto deberán ir más allá del solo asociacionismo, para convertirse en constructivismo de los aprendizajes, en búsqueda de una escuela inclusiva y de que cada persona sea más participativa en la construcción del conocimiento.

La función que el aprendizaje y la educación tienen en la sociedad es una constante que a lo largo de los años, se ha ido poniendo de manifiesto con más fuerza a través de diversos medios y adoptando diferentes formas; por ello no es de sorprender que las TD cumplan ahora un rol dinamizador entre la educación y la sociedad (Carneiro, Toscano y Díaz, 2009).

Las aplicaciones educativas de las TD deben ser integradas a nivel educativo como en un sentido más amplio, respecto a lo socioeconómico, relacionados ambos con conceptos que van mucho más allá de la perspectiva escolar (Benavides y Pedró, 2007); en tal sentido, dichos autores evidencian la existencia de cuatro ejes comunes, donde las TD cumplen una función vital; el primero de ellos apunta al aporte que tienen sobre el desarrollo económico de todo país; el segundo es el de equidad y justicia social; el tercero es la de cambio pedagógico y el cuarto eje es el de calidad en el aprendizaje.

La incorporación de las TD en el ámbito educativo es considerada fundamental para la consecución de todo proyecto pedagógico, por cuanto potencian el trabajo colaborativo y el desarrollo de habilidades adaptativas. Por otro lado, la idoneidad del docente es un aspecto fundamental en el buen desarrollo de un proceso formativo, aspectos que se ven reflejados en la existencia de un aprendizaje de calidad. Por último, cabe anotar que la inversión en equipos y la capacitación de los profesores, resultan relevantes y las instituciones de educación son conscientes tanto de la existencia de esta necesidad, como del impacto de dicha inversión (Baltodano y Quesada, 2016).

Considerando lo anteriormente descrito, los escenarios en educación han tenido que ajustarse a los cambios que impone la sociedad. En este sentido, son pertinentes nuevos enfoques educativos y estrategias metodológicas conforme a la realidad, vinculadas y consecuentes con las exigencias del estudiantado. Se hacen necesarios estos ajustes, ya que los procesos de enseñanza y aprendizaje deben ir encaminados a la apropiación de conocimientos significativos y perdurables en el estudiantado; problematizando la realidad circundante, para que los objetos de estudio propuestos en el currículo nacional tengan sentido práctico. Todo lo anterior, permite establecer la necesidad para la promoción de las TD como herramientas para facilitar el desarrollo del currículo. Así lo describe la Organización de las Naciones Unidas para la Educación, la Ciencia y la Cultura (2013), cuando afirma que las TD: 
...pueden contribuir al acceso universal a la educación, la igualdad en la instrucción, el ejercicio de la enseñanza y el aprendizaje de calidad y el desarrollo profesional de los docentes, así como a la gestión dirección y administración más eficientes del sistema educativo (párr. 1).

Según la cita anterior, al existir la necesidad de planificar e implementar innovaciones educativas con el uso de las TD en los procesos de enseñanza y aprendizaje, son innegables las expectativas existentes para flexibilizar y transformar el papel y las creencias de los docentes hacia el proceso educativo.

\section{PROPOSICIÓN}

La integración de las TD, propone el reconocimiento del papel activo de los estudiantes dentro del proceso, además, de la concientización de la complejidad de los contextos educativos y de la rapidez con la que evoluciona la sociedad actual. En este sentido y considerando los aspectos descritos, se tienen como objetivos de los proyectos colaborativos con el uso de TD:

- "Propiciar ambientes de aprendizaje colaborativo entre educadores y entre estudiantes.

- Favorecer el desarrollo de experiencias de aprendizaje a partir de las cuales, los profesores revisen y reflexionen su práctica.

- Potenciar el aprendizaje en red y el intercambio intercultural entre estudiantes de distintos contextos geográficos" (Baltodano, 2014, p. 5).

Un proyecto colaborativo se define como, una metodología didáctica que tiene como finalidad organizar el trabajo colaborativo entre estudiantes y profesores, para la realización de actividades educativas que coadyuven al abordaje integral de diversos problemas del entorno socioeducativo. Según Serrano (2010), los proyectos colaborativos propician “...la generación de conocimiento. En ellos se realizan planteamientos en la que los estudiantes conformados en grupos de trabajo, obtienen productos o materiales elaborados por ellos mismos..." (p. 1), promoviendo así aprendizajes significativos por medio de las interpretaciones que los individuos hacen de su entorno.

Por otro lado, cabe denotar que existe una relación entre el aprendizaje y el trabajo colaborativo, puesto que los participantes en el proceso de aprendizaje se afectan mutuamente, al generar propuestas conjuntas y expectativas relacionadas con su contexto sociocultural. Según Calzadilla (2002), el aprendizaje generado mediante la colaboración "facilita el desarrollo de aquellos procesos cognitivos, como la observación, el análisis, la capacidad de síntesis, el seguir instrucciones, comparar, clasificar, tomar decisiones y resolver problemas, en los que la interacción enriquece los resultados y estimula la creatividad" (p. 5).

Según eso, el aprendizaje colaborativo se fundamenta pedagógicamente en la teoría cognoscitiva de Piaget, ya que según este autor las estructuras cognoscitivas se modifican si intervienen factores como la maduración, la experiencia y la trasmisión social, entre otros (Calzadilla, 2002), aspectos que sin duda son favorecidos en ambientes de aprendizaje colaborativos con el uso de TD. Asimismo, mediante el desarrollo del aprendizaje colaborativo los participantes son responsables de generar andamiajes y estructuras propias que les permiten apropiarse del conocimiento (Vygotsky, 1979). Por ello, el aprendizaje colaborativo se sitúa también dentro de la teoría constructivista del aprendizaje, ya que se concibe "... como proceso de socio-construcción que permite conocer las diferentes perspectivas para abordar un determinado problema, desarrollar tolerancia en torno a la diversidad y pericia para reelaborar una alternativa conjunta [de aprendizaje]" (Calzadilla, 2002, p. 3). 
En relación con lo anterior, los proyectos colaborativos incentivan la creación de entornos personales de aprendizaje en los cuales se espera que quienes trabajan en determinado proyecto definan los objetivos, las metas y la planeación, no obstante dicha tarea "...requiere liderazgo, motivación, reflexión, organización y participación" (Barrera, 2008, p. 69), asimismo el acceso al conocimiento que proviene de objetos de aprendizaje y de otras personas (Castañeda y Adell, 2013).

Para iniciar un proyecto colaborativo es necesario conformar los equipos de trabajo. Según Barrera (2008) los equipos de trabajo son "...unidades especiales encargadas de cumplir con metas concretas" (p. 70). Los equipos de trabajo existen generalmente de manera transitoria mientras existan las tareas o iniciativas que dieron origen al proyecto. En relación con la duración del proyecto dependerá de la iniciativa de los participantes y podrá variar de acuerdo con las necesidades de estos (Instituto Latinoamericano de Comunicación Educativa citado por Baltodano, 2014).

En referencia a la cantidad de participantes en los equipos de trabajo, no existe una norma definida para establecer la cantidad, sin embargo, es necesario garantizar que la cantidad de personas no se convierta en un elemento disruptivo durante el desarrollo del proyecto. Una vez definida la cantidad de participantes y constituidos formalmente los equipos, se inicia la comunicación con la contraparte, generalmente los profesores y estudiantes utilizan canales como: el correo electrónico, las redes sociales Twitter, Facebook, Instagram u otras herramientas o redes sociales para saludarse, intercambiar fotos u otras actividades relacionadas con el proceso de contacto inicial. El proceso de interacción en equipo conlleva a varias etapas, a saber: inicial, luna de miel, integración, conflicto y madurez.

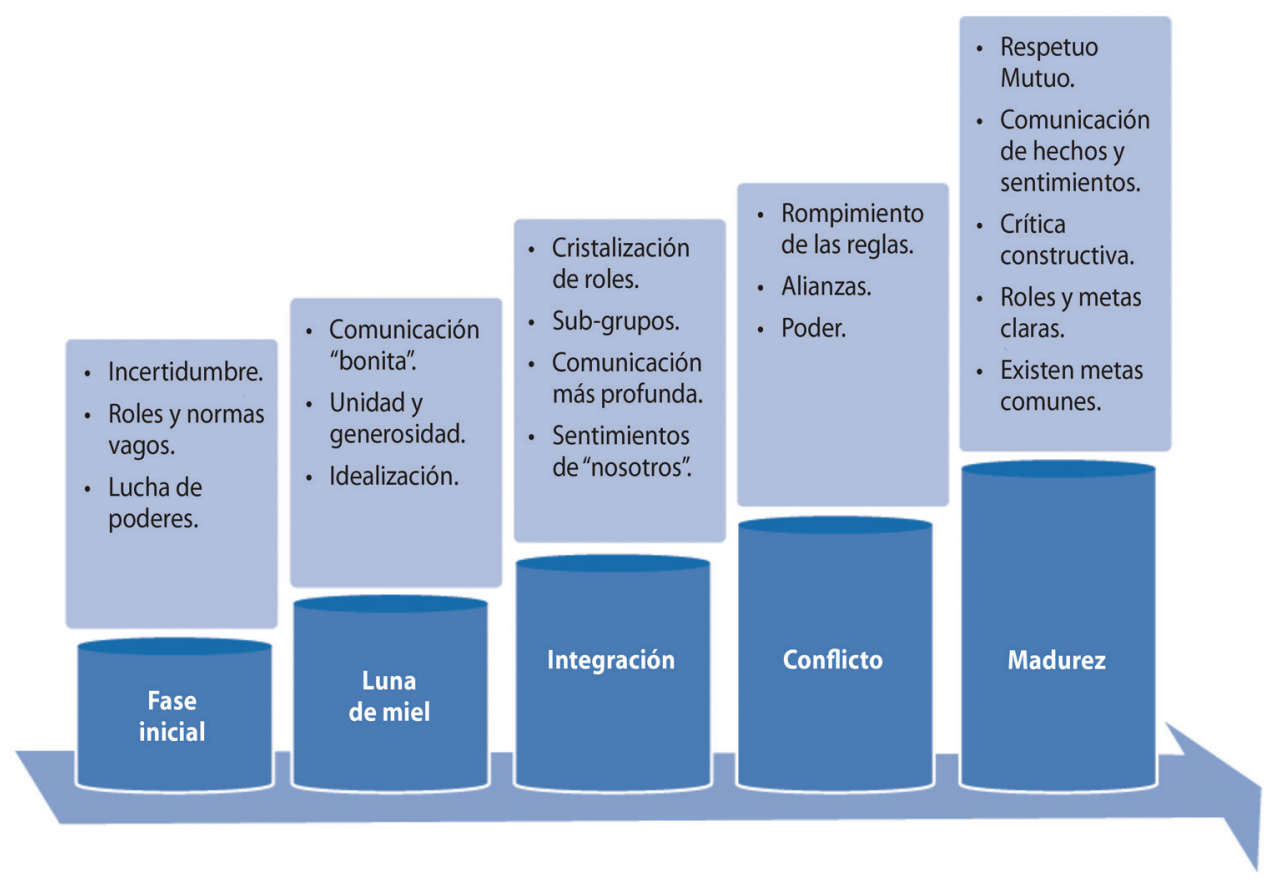

Figura 1. Etapas del proceso de interacción durante el trabajo colaborativo. Fuente: Elaboración propia a partir de Laureate International Universities (2013). 
Conforme avance la elaboración de proyectos colaborativos, que tengan como eje trasversal el uso de las TD será necesario compartir, publicar y circular distintos asuntos y producciones. Para ello, son imprescindibles los espacios compartidos de colaboración en línea, así como, los blogs, las páginas web, una revista electrónica o una red social, serán herramientas muy útiles para su creación.

Los proyectos colaborativos deben ajustarse de manera pertinente al estudiantado e invitar a trabajar con orientaciones tecnológicas, abordando temáticas curriculares y contextuales, sobre la conceptualización y enfoque colaborativo. Al respecto, Fuentes y Ballestero (2008), afirman que “...los estudiantes... serán, en última instancia, las personas que extenderán todo el conocimiento adquirido al campo de la realidad, de su propio entorno" (p. 3). Permitiendo así, el desarrollo de prácticas educativas creativas e innovadoras, fomentando el aprendizaje colaborativo, reduciendo los procesos rutinarios y compartiendo conocimientos con otros de manera virtual.

Todo proyecto pedagógico se debe asumir como un escenario propicio para hacer efectiva la colaboración. Asimismo, ser propulsor de un proceso de investigación, que involucre observaciones, cuestionamientos, documentación, formulación de hipótesis, conclusiones y recomendaciones (Thomas, 2000), articulando las propuestas con la realidad comunal e institucional, tal y como lo muestra la siguiente figura.

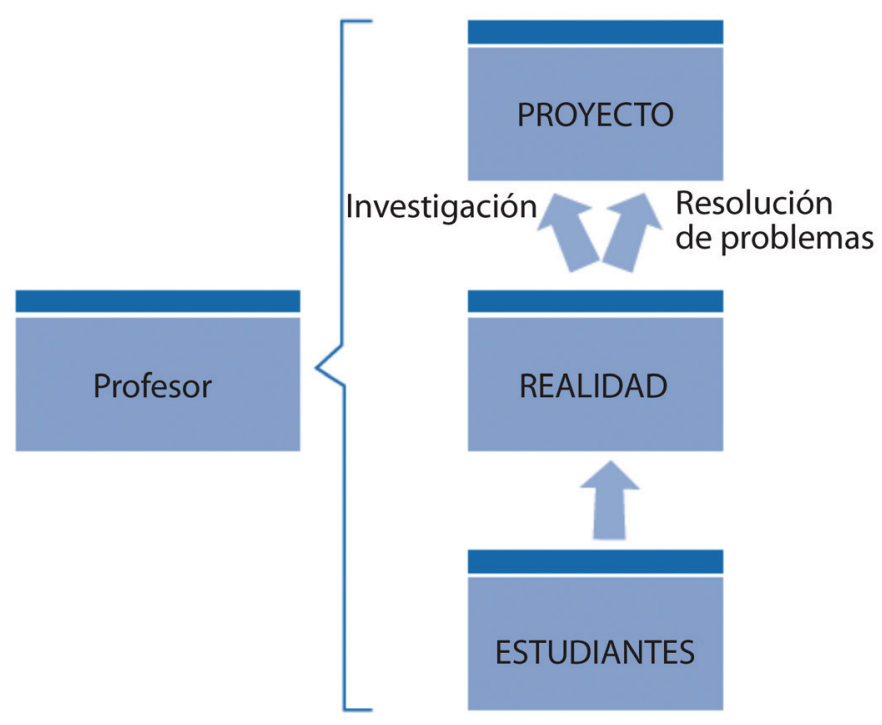

Figura 2. Escenario de colaboración de los proyectos pedagógicos. Fuente: Elaboración propia.

La implementación del proyecto colaborativo actuará como escenario para comprender y transformar aspectos de la realidad sociocultural, mediante procesos de investigación, resolución de problemas y elaboración de productos creativos, que realizan los estudiantes con la orientación del profesor. Durante la planificación, el desarrollo y la implementación de los proyectos, la investigación se convierte en un eje transversal, debido a que favorece la construcción de conocimientos, la socialización de ideas, la comunicación asertiva y el trabajo colaborativo derivados de la interacción grupal.

La elaboración del proyecto colaborativo conlleva a cinco fases, a saber: la preparación, planificación, implementación, evaluación y divulgación. La preparación permite pensar el proyecto, definir sus alcances y definir todas las condiciones para su desarrollo. En esta etapa, se concreta la idea inicial, producto del intercambio entre los participantes y las condiciones iniciales. 
En un segundo orden, la planificación conduce a la definición del tema específico del proyecto, la pregunta problema, la justificación, los objetivos, la metodología. Por otra parte, como tercera fase se encuentra la implementación, que consiste en la ejecución de todas las acciones planeadas y distribuidas entre los participantes, contempla el intercambio, la indagación y el desarrollo de los objetivos.

Dadas las condiciones que anteceden, la fase cuatro es la evaluación, que es un componente trasversal desde el proceso de planificación hasta la divulgación, generalmente, se da seguimiento y evidencia el grado de avance mediante bitácoras que los participantes elaboran cada vez que interactúan.

Por último, se tiene la fase de comunicación de los resultados, la cual posibilita la reflexión y el intercambio de los conocimientos adquiridos en todas las fases del proceso; además de que permite formalizar la presentación de los resultados y productos obtenidos. Esta etapa incluye el informe final, la publicación de este y la difusión del proyecto colaborativo.

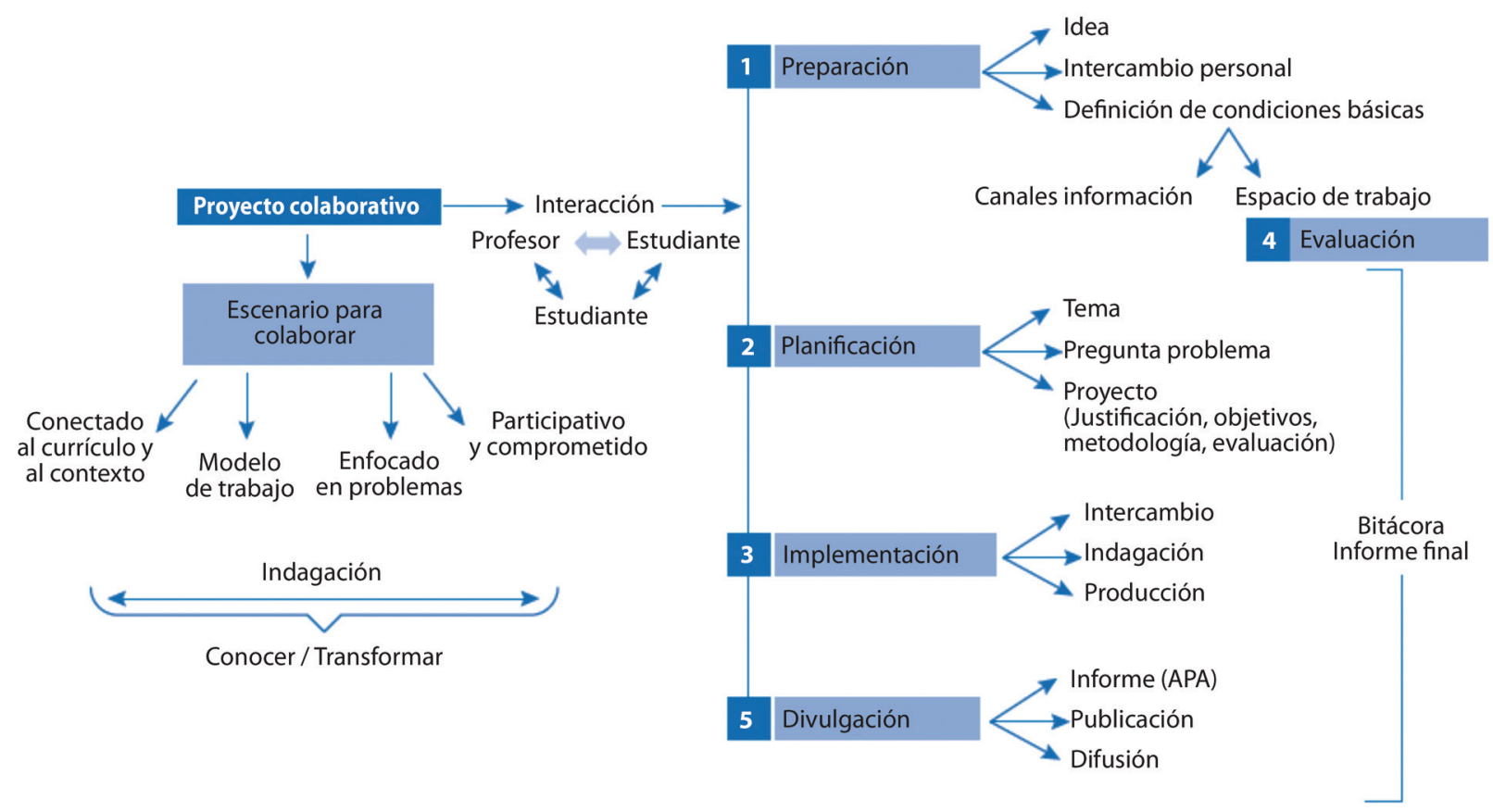

Figura 3. Propuesta para el desarrollo de proyectos colaborativos. Fuente: Elaboración propia.

A partir de la propuesta anterior, los profesores se enfocan en la promoción de un ambiente participativo, reflexivo, analítico y crítico que permita modelar las destrezas comunicacionales esperadas y favorecer, la motivación hacia un compromiso dirigido a la construcción de aprendizajes significativos. Al asumir un compromiso por su propio proceso de aprendizaje, los participantes de los proyectos colaborativos se comprometen con los procesos de atención, reflexión pedagógica, diálogo y en la elaboración creativa de insumos que permitan la consecución de los objetivos propuestos. En el desarrollo de proyectos colaborativos, se espera la puesta en práctica de los cinco elementos, a saber: interdependencia positiva, promoción de la interacción, responsabilidad individual, interacción positiva y habilidades y destrezas de trabajos grupales. 
Los miembros de un grupo persiguen un objetivo común y comparten recursos de información. No compromete a los miembros en forma competitiva y el resultado es más que las contribuciones individuales agregadas.

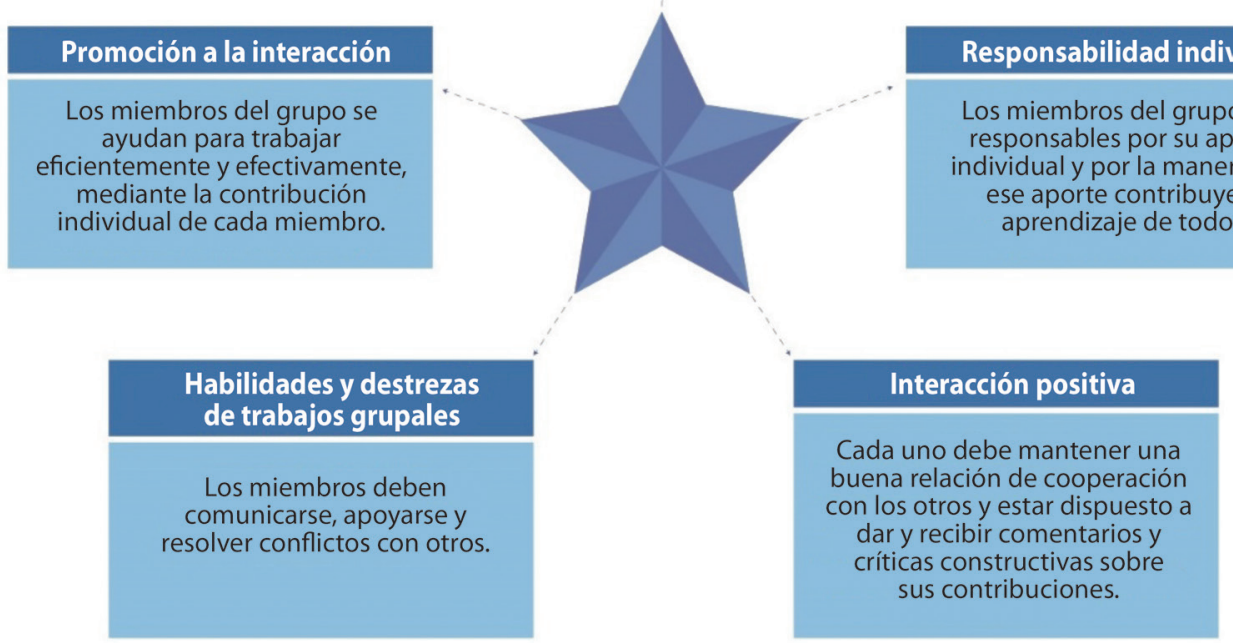

Figura 4. Elementos del aprendizaje promovidos en proyectos colaborativos. Fuente: Elaboración propia a partir de Johnson, Johnson y Holubec (1999).

Mediante estos elementos, el trabajo práctico y la reflexión teórica o empírica permitirán el logro del objetivo general y los objetivos específicos. Los participantes evidencian dichos elementos durante el desarrollo de proyectos colaborativos, posibilitando así, procesos y prácticas reflexivas que coadyuvan con el crecimiento personal.

\section{ARGUMENTOS PARA LA DISCUSIÓN}

El trabajo colaborativo propicia el acercamiento entre los individuos que comparten metas y problemas comunes, asimismo coadyuva al establecimiento de una visión integral de la realidad ya que en él, convergen diferentes perspectivas y posiciones de los participantes. En relación con lo anterior, el trabajo colaborativo como estrategia pedagógica promueve el desarrollo de capacidades, destrezas y habilidades que permiten una mejor compresión del contexto sociocultural y el planteamiento de propuestas para la solución de problemas sociales.

En el marco del constructivismo social, el trabajo colaborativo conecta a los individuos con su entorno y con las diversas alternativas para solución de problemas. Por otro parte, la conformación de equipos de trabajo juega un papel importante en la generación de conocimientos, así como también, en el aprovechamiento de las TD como recursos didácticos que facilitan la profundización y el desarrollo cognitivo, socioafectivo y psicomotor de los estudiantes. Por lo antes descrito, el logro de los aprendizajes dependerá no solamente de las aptitudes y actitudes individuales, sino también de la intensidad con que los participantes establezcan la colaboración (Román, 2004).

Según lo anterior, son innegables los beneficios que proporcionan las TD para acrecentar los niveles de colaboración e interacción de los individuos, tanto de manera asincrónica como sincrónica. Las diversas 
posibilidades para la creación de canales de comunicación, la innovación educativa y el desarrollo de la creatividad hacen de las TD excelentes aliadas para romper el modelo de enseñanza tradicional, así como barreras idiomáticas, temporales y geográficas. No obstante, para sacar máximo provecho de ellas, es necesario garantizar condiciones óptimas de infraestructura tecnológica, conectividad y soporte técnico.

En el marco de las consideraciones anteriores, las TD coadyuvan al desarrollo de procesos de enseñanza y aprendizaje acordes con las necesidades comunitarias y escolares. Lo anterior, cobra sentido cuando se establecen mecanismos que permiten el crecimiento individual y colectivo de los actores curriculares. El centro educativo como dinamizador de los aprendizajes, deberá propiciar espacios colaborativos que favorezcan el desarrollo del currículo, pero además que incentiven el crecimiento colectivo. Todo lo anterior, posibilitaría la socialización e interacción entre los miembros de la comunidad educativa, promoviendo así el desarrollo humano y la construcción colectiva del conocimiento (Instituto Tecnológico y de Estudios Superiores de Monterrey, s.f).

El planteamiento anterior, requiere de ambientes colaborativos y comunidades de práctica donde los docentes puedan interactuar y reflexionar en relación con la planificación, desarrollo e implementación de los proyectos colaborativos. En este sentido, las TD proveen de una serie de recursos educativos abiertos que coadyuvan a la planificación de actividades de mediación, el seguimiento y la evaluación de proyectos. Cabe agregar, que otro elemento para garantizar el éxito de un proyecto colaborativo, lo constituye el grado de motivación que se logre cultivar en los estudiantes y las estrategias que utilice el docente para la conformación de los grupos de trabajo.

\section{SÍNTESIS Y REFLEXIONES FINALES}

Los proyectos colaborativos surgen como iniciativas escolares promovidas por los actores curriculares en los distintos ámbitos del sistema educativo. Una característica importante de este tipo de iniciativas pedagógicas se asienta en el hecho, de recolectar las expectativas o inquietudes de quienes participan y traducirlas en aprendizajes significativos.

La incorporación de TD y redes sociales permite el desarrollo de proyectos colaborativos entre diferentes centros educativos sin importar la ubicación de estos y la temporalidad. Asimismo, benefician el acercamiento con la información en centros de documentación, bases de datos científicas a través de la web de páginas especializadas, en las temáticas seleccionadas en cualquier momento y lugar. De la misma forma, las TD permiten que las responsabilidades y los alcances del proyecto por trabajar no se circunscriban a un contexto específico, sino que por medio del internet, se compartan en diferentes entornos.

Un elemento fundamental para el desarrollo de proyectos pedagógicos con el apoyo de las TD requiere el que los centros educativos cuenten con recursos básicos como conectividad y equipos suficientes para trabajar las actividades; se debe adoptar la decisión de implementación en infraestructura tecnológica en forma inmediata, que permita alcanzar los objetivos previstos en los proyectos.

Asimismo, la formación docente y los procesos de capacitación son fundamentales para garantizar el éxito en proyectos pedagógico que incorporan las TD como herramientas de aprendizaje. En este sentido, se ha podido percibir en la evaluación de los proceso de formación continua, que las acciones formativas desarrolladas hasta el momento, no han sido suficientes para garantizar la incorporación de las TD como apoyo al currículo, sin embargo, se está avanzando hacia el nivel de integración deseado.

Otro elemento fundamental es el seguimiento y acompañamiento pedagógico que necesitan los profesores involucrados, esto debido a carencias básicas en el uso de TD, lo que lleva a plantear que no puede hablarse de una integración propiamente, si aún faltan elementos de formación. Por otro lado, se puede 
apoyar a los docentes, generando redes de apoyo que se requieren para llegar al nivel de transformación de las prácticas pedagógicas, gracias a la colaboración y al trabajo autogestionado.

Aunque los profesores emplean los servicios como: el correo electrónico, las redes sociales, y algunos de ellos, ya están avanzando en la creación de recursos digitales para el aprendizaje. Sigue estando presente la necesidad de que cada proyecto contemple diversas iniciativas para el uso de TD, las cuales pueden irse potencializando cada vez más y de manera paulatina en las prácticas pedagógicas

Por último, uno de los retos fundamentales del Departamento de Investigación, Desarrollo e Implementación para la puesta en marcha de proyectos pedagógicos con uso de TD como apoyo al currículo, será lograr un mayor alcance en la cobertura de la infraestructura digital por medio de un equipamiento adecuado y a la vez, dar un paso más allá de la mera alfabetización digital de los actores involucrados, partiendo de la búsqueda de nuevas formas de comunicación y colaboración entre los integrantes de las comunidades de aprendizaje.

\section{REFERENCIAS}

Asociación Nacional de Universidades e Instituciones de Educación Superior. (2004). Documento Estratégico para la Innovación en la Educación Superior. Recuperado de https://comitecurricularsistemas.wikispaces.com/file/view/INNOVACION_EN_LA_EDUCACION_SUPERIOR_ANUIES_ MAYO+2004.pdf

Baltodano, M. (2014). Aulas en Red: Un modelo para aprender en forma colaborativa. San José: Ministerio de Educación Pública.

Baltodano, M. y Quesada A. (2016). Ideas didácticas para el aprovechamiento de las tecnologías digitales como recursos de aprendizaje. San José: Ministerio de Educación Pública.

Barrera, M. (2008). Cómo elaborar proyectos urgentes. ( $2^{\mathrm{a}}$ ed.). Caracas: Quirón S. A. y Sypal.

Benavides, F. y Pedró, F. (2007). Políticas Educativas sobre Nuevas Tecnologías en los países

Iberoamericanos. Revista Iberoamericana de Educación, (45), 19-69. Recuperado de: http://www.rieoei. org/rie45a01.pdf

Calzadilla, M. (2002). Aprendizaje Colaborativo y Tecnologías de la Información y la Comunicación. Revista Iberoamericana de Educación, 1-11. Recuperado de http://rieoei.org/tec_edu7.htm

Carneiro, R., Toscano, J. C. y Díaz, T. (2009). Los desafíos de las TIC para el cambio educativo.

Madrid: Colección Metas Educativas, OEl/Fundación Santillana. Recuperado de http://iec-peru.org/pdf/ cambio_educativo.pdf

Castañeda, L. y Adell, J. (Eds.). (2013). Entornos Personales de Aprendizaje: claves para el ecosistema educativo en red. Alcoy, España: Marfil.

Claro, M. (2010). La incorporación de tecnologías digitales en educación. Modelos de identificación de buenas prácticas. Santiago de Chile: CEPAL.

Fuentes, M. y Ballestero, J. (2008). Aulas Hermanas un Trabajo Entre Pares Utilizando Entornos Virtuales. Congreso Virtual Iberoamericano de Calidad en Educación a Distancia. Recuperado de http:// eduqa2008.eduqa.net/eduqa2008/images/ponencias/eje_tematico_5/5_68_Aulas_hermanas_

Fuentes_Ballestero_.pdf

Instituto Tecnológico y de Estudios Superiores de Monterrey. (s.f). Las estrategias y técnicas didácticas en el rediseño: aprendizaje colaborativo. Recuperado de http://sitios.itesm.mx/va/dide2/tecnicas_didacticas/ac/Colaborativo.pdf 
Johnson, D., Johnson, R. y Holubec, E. (1999). El aprendizaje cooperativo en el aula. México D.F.: Paidós Mexicana S.A. Recuperado de http://cooperativo.sallep.net/El\%20aprendizaje\%20cooperativo\%20en\%20el\%20aula.pdf

Laureate International Universities (2013). Teamwork in Project-Based Learning. Baltimore, USA: Laureate Network Products \& Services.

Organización de las Naciones Unidas para la Educación la Ciencia y la Cultura. (2013). Las tecnologías de la información y la comunicación (TIC) en la educación. Recuperado de http://www.unesco.org/new/ es/unesco/themes/icts/

Román, P. (2004). Los entornos de trabajo colaborativo y su aplicación en la enseñanza. En Cabero, J. y Romero, R. (Coords.). Nuevas tecnologías en la práctica educativa, 213-254. Granada: Arial Ediciones, S.L. Recuperado de http://blogs1.uoc.es/racev/files/2013/02/2004entornosdetrabajocolaborativoy aplicacionenseanza-arial.pdf

Serrano, M. (2010). "Diseño de Proyectos Colaborativos". Revista e-Formadores, 1-9. Recuperado de http://red.ilce.edu.mx/sitios/revista/e_formadores_pri_10/articulos/angeles_serrano_feb2010.pdf

Thomas, J. (2000). A review of research on project-based learning. Recuperado de http://www.bobpearlman.org/BestPractices/PBL_Research.pdf

Vygotsky, L. S. (1979). El desarrollo de los procesos psicológicos superiores. Madrid: Grijalbo. 
